\title{
Experimental Analysis of Heat Transfer Augmentation in Double Pipe Heat Exchanger using Tangential Entry of Fluid
}

\author{
Hanumant Jagdale, Subhash Lahane \\ (Department of Mechanical Engineering, MIT Aurangabad, Dr. BAMU Aurangabad, Maharashtra, India)
}

\begin{abstract}
In this work, the effect of heat transfer enhancement of swirl generator with tangential injection of fluid was examined. The five tangential injectors are placed such that they are equidistance along the length of pipe to improve the efficiency of heat transfer in concentric pipe heat exchanger the injectors are designed and placed such that it creates tangential flow in the pipe. The experiment performed for water as working fluid, hot water flowing through inner tube of material mild steel with inner diameter (ID) $16 \mathrm{~mm}$ and outer diameter $(O D) 21 \mathrm{~mm}$ and cold water flowing through annulus space to generate swirling motion. The outer tube of material UPVC (un-plasticized polyvinyl chloride) having ID $35 \mathrm{~mm}$ and OD $42 \mathrm{~mm}$. The experiment was performed for different Reynolds number ranging from 2200- 4600 for parallel and counter flow configurations. It is observed from the experimental results that the heat transfer rate increased with increase in Reynolds number. Heat transfer rate was increased about $20 \%$ to $70 \%$ with tangential injector type swirl generator than the heat exchanger without tangential injectors. It was also observed that the maximum heat transfer coefficients $(1613 \mathrm{~W} / \mathrm{m} 2 \mathrm{~K}$ ) could be achieved at Reynolds number of 4600 for all (five) nozzles opening with 70 $\%$ increase in heat transfer.
\end{abstract}

Keywords - Tangential entry of fluid, swirl flow, heat transfer augmentation, nozzles

\section{INTRODUCTION}

Heat transfer is widely used in many applications in heat exchangers, chemical process, gas and oil production, air condition, automotive or food industries. Modern engineering and technology is moving constantly upward on the temperature scale and subsequently the concept of augmentation of heat transfer has derived the attention of many scientists and engineers in the past few decades [1]. A general consideration in designing a heat exchanger is to make it compact, i.e., increase the overall heat transfer coefficient. Another consideration is reducing the temperature of driving forces which enhances the second law efficiency and decreases entropy generation. Over the past decades, several methods have developed and extensively applied to heat exchangers [2]. The double pipe heat exchangers are the most important device used in industrial and engineering applications. The economic and energy considerations continuously motivate investigators to search for efficient methods of heat transfer. There are different heat transfer augmentations methods available to increase the heat transfer rate and make instrument compact and incorporated into heat exchanger are passive, active and compound techniques [3]. Passive technique is used surface or geometrical modifications to the flow by incorporating inserts or additional devices. Higher heat transfer coefficients achieved by disturbing the flow behavior which also leads to increase in the pressure drop. Passive techniques hold the advantage over the active techniques as they do not require any direct input of external power [3-4]. This technique includes treated surfaces, rough surfaces, extended fins, swirl flow device, coiled tubes etc. Active techniques require external power to disturb the flow and improvement in heat transfer rate. This technique finds limited application because of the need of external power in many practical applications [4]. This technique includes mechanical aids, surface vibration, fluid vibration, injection, jet impingements etc. In compound techniques, there is use of one or more than one of the above mentioned techniques in combination to improve the performance of a heat exchanger [4]. Active and compound methods are rarely used because of their complexity \& cost [5]. The swirl flow is one of the techniques that have been used successfully in the past to augment heat transfer [6]. The heat transfer augmentation along with swirl in the flow was proposed by Kreith and Margolis [7]. In this concept, tangential injection was used and the vortex motion maintained by repeated addition of fluid through appropriately spaced injection holes for any desired distance. Swirl flows result from an application of a spiral motion, a swirl velocity component (also called as 'tangential' or 'azimuthal' velocity component) being imparted to the flow by the use of various swirl-generating methods. Many researchers have studied the heat transfer characteristics of swirl flows by using various swirls. Generally, the swirling pipe flows are classified into two types: (i) Continuous swirl flows, which maintain their characteristics over entire length of test section;

$5^{\text {th }}$ National Conference RDME 2016, 10-11 $1^{\text {th }}$ March 2016. 

entry of fluid

and (ii) decaying swirl flows [8]. Gupta et al. [9] classified the method of generating swirl into three main categories- (i) tangential entry, (ii) guide vanes and (iii) direct rotation. The tangential entry of the fluid into a duct stream can be achieved by a single tangential inlet duct (circular or rectangular at different angles to the pipe axis) or more than one tangential entry, tangential inlet nozzles, tangential machined slots and tangentially drilled slots. The swirl can be produced in the flow by tangential of the fluid by single tangential entry or the combination of axial plus tangential entry. Dhir, et al. [10] experimentally determined augmentation of heat transfer with air as the test fluid and Reynolds numbers ranging from 15000 to 58000 . The air was injected tangential to the inner walls of the heat exchanger tubes through square edged injectors extending perpendicular from the tube surface. The net augmentation of heat transfer, at constant pumping power, was between 3\% and $14 \%$ depending on the momentum ratio. It was also found that the effectiveness of the system is highly dependent on the ratio of the rates of tangential to total momentum fluxes. The two measure mechanisms of augmentation are observed- i) high maximum axial velocity near the wall produces higher heat flux from the wall, ii) high turbulence level in the middle region of tube improves mixing and thus, the rate of heat transfer [11]. Chang and Dhir [11] carried out an experimental study for the heat transfer augmentation for a turbulent flow with tangentially injected swirl flows in vertical tubes. It was found that the momentum flux ratio is the most effective parameter that enhances heat transfer while Reynolds number affects it weakly and the effect of the fluid Prandtl number is very weak. Also, the injector tube diameter and number of injectors do not affect the heat transfer enhancement. The effect of injection include swirl flow on single and two phase heat transfer using water as a working fluid was investigated by Guo and Dhir [6]. It was found that, on a constant pumping power basis, a net enhancement of about $20 \%$ could be achieved experimentally at a momentum flux ratio of about 9.6. The different parameters of swirling flow generated by injection were studied experimentally by Akpinar et al. [12-13] and Çakmak and Yıldız [14]. They studied the effect of holes diameter, holes number and angle of injection on the heat transfer rate and pressure drop. They found that, using injector to create swirling flow enhances heat transfer rate but it increases the pressure drop and requires more pumping power.

\section{EXPERIMENTAL DETAILS}

Experiment was performed for both parallel flow and counter flow for different values of Reynolds number from 2000 to 5000 and for different nozzles openings. In this experiment hot water was passed through inner tube, while cold water passed through annulus space between inner and outer tube. Hot water was achieved by gas geyser provided to the experimental setup as shown in Fig. 1.
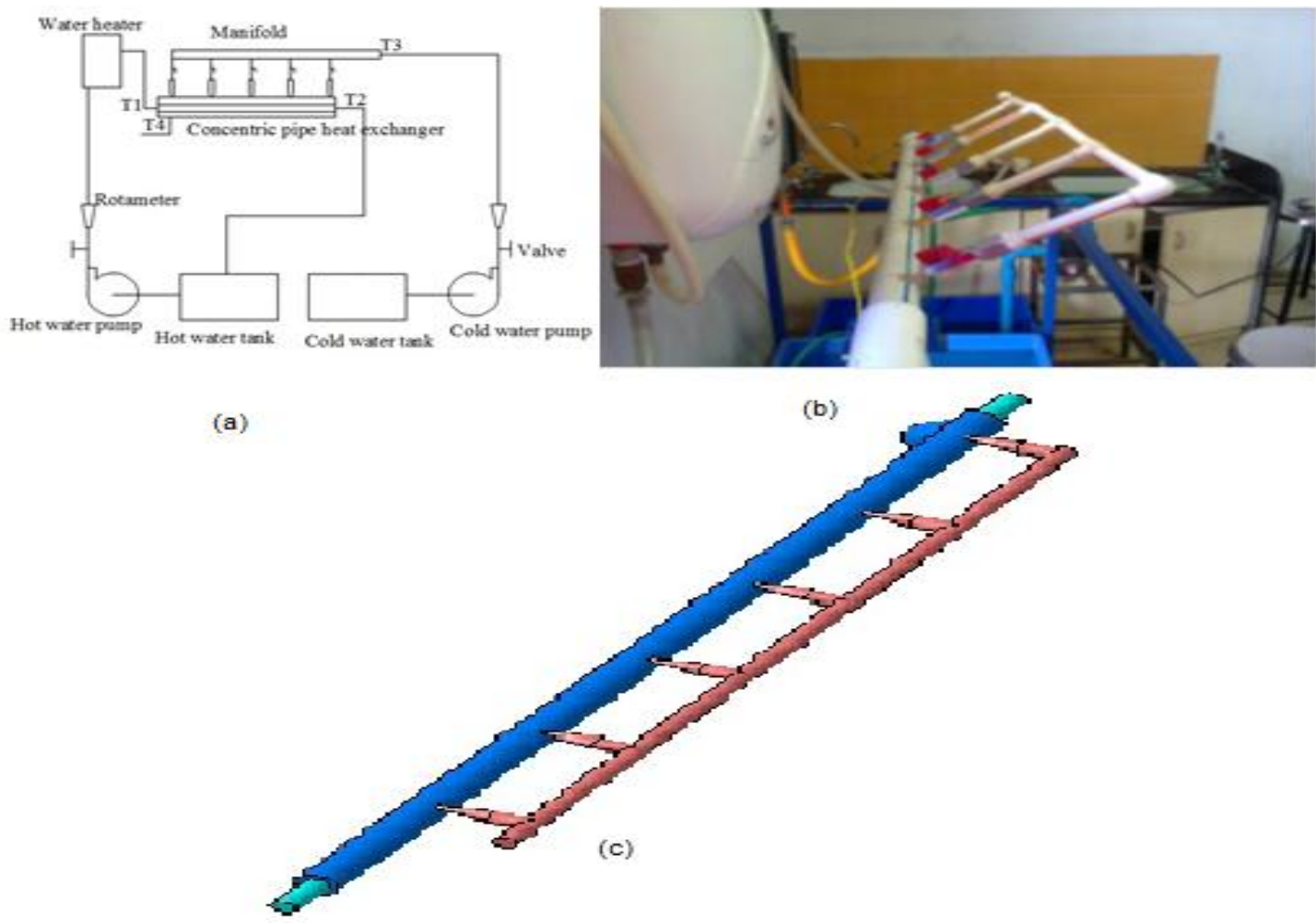

Fig.1. (a) Layout of experimental set-up, (b) Working set-up (c) 3-D view of tangential injection tube of double pipe heat exchanger with tangential entry. 
The mass flow rate of hot water keeping as constant i.e.300 LPH and that of cold water was varied from $300 \mathrm{LPH}$ to $600 \mathrm{LPH}$ in the step of $50 \mathrm{LPH}$ and simultaneously temperature of inlet and outlet of hot and cold water were recorded. Temperatures of cold water and hot water inlet and outlet were measured with the help of thermocouples. Five nozzles are fitted tangential to the axis of pipe equidistance along the length of tube such that they are tangentially perpendicular to the length of pipe. Nozzles are opened (consecutive opening and closing) one by one and the temperatures at the inlet and outlet of cold and hot water are measured. The layout of experimental setup and working setup of tangential entry nozzles are shown in Fig. 1.

\section{MATHEMATICAL CALCULATIONS}

In concentric heat exchanger the heat given by hot fluid is expressed by Equation 1 and it is the function of mass of hot fluid, specific heat and temperature difference.

$$
Q_{h}=m_{h} C_{p}\left(T_{h o}-T_{h i}\right)
$$

The heat received by cold fluid is expressed by Equation 2 and it is the function of mass of cold fluid, specific heat and temperature difference.

$$
Q_{c}=m_{c} C_{p}\left(T_{c o}-T_{c i}\right)
$$

The average heat transfer rate for hot and cold fluid is calculated using Equation 3 by calculating the average of heat given by hot fluid and heat received by cold fluid.

$$
Q_{\text {avg }}=\frac{Q_{h}+Q_{c}}{2}
$$

The overall heat transfer coefficients is calculated by using Equation 4 and it is the function of average heat transfer, logarithmic mean temperature difference (LMTD) and outer surface area.

$$
U_{o}=\frac{Q_{\text {avg }}}{A_{o} L M T D}
$$

Reynolds number can be calculated by using Equation 5 and which is the function of inertial force to the viscous force.

$$
\operatorname{Re}=\frac{\rho V d_{h}}{\mu}
$$

The thermo physical properties of water which is used to calculate Reynolds number is determined at mean temperature calculated using Equation 6.

$$
T_{\text {avg }}=\frac{T_{i}+T_{o}}{2}
$$

\section{RESULTS AND DISCUSSION}

The experimental value of heat transfer coefficient is determined by plotting Wilson chart [15-16]. The Wilson chart is plotted for variation of $1 /(\operatorname{Re})^{0.8}$ with $1 /$ Uo as shown in Fig. 2. The ' $y$ ' intercept as " $k$ " (fouling resistance) taken from the Wilson chart and heat transfer enhancement is calculated by using Equation 7.

$$
\frac{1}{h_{\text {exp } t}}=\frac{1}{U_{o}}-k
$$




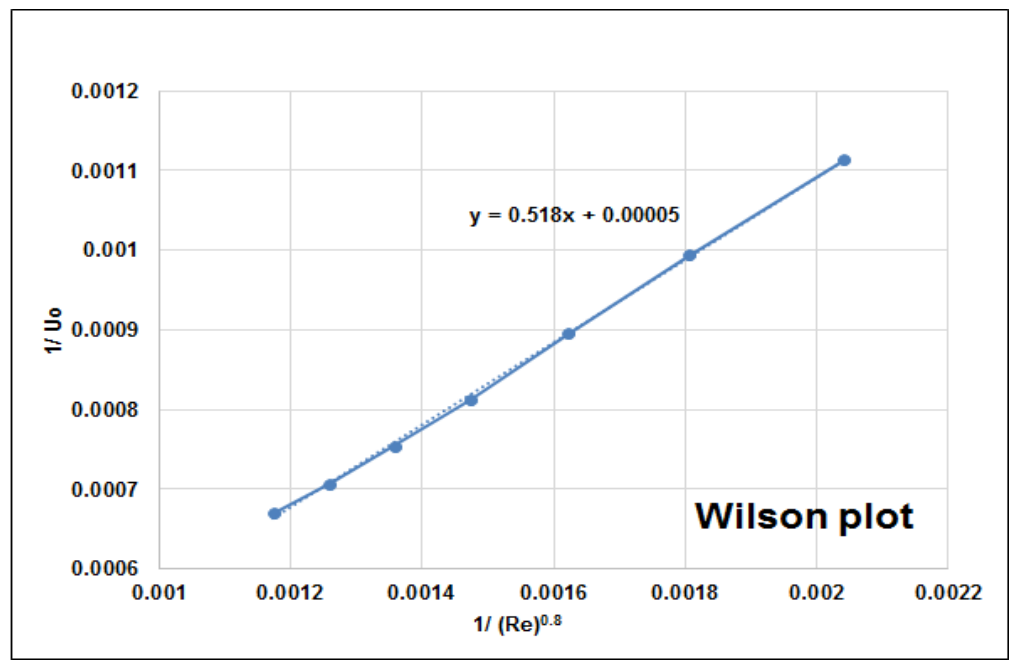

Fig.2. Wilson plot

The experiment was performed for different Reynolds number for different nozzle openings and the results obtained for parallel and counter flow models for heat transfer coefficient are discussed in this section. Heat transfer coefficient results for plain tube and tube with tangential entry for parallel flow and counter flow model are obtained for range of Reynolds number from 2000-5000.

The comparison of the plain pipe and pipe with tangential entry of fluid with different nozzle openings is shown in Fig. 3. It is seen that $h_{\text {expt }}$ increases with increase in Reynolds number for both the configurations. For plain pipe $h_{\text {expt }}$ value is varies from $457-772 \mathrm{~W} / \mathrm{m}^{2} \mathrm{~K}$ for range of Reynolds number 2000-5000. As compared to tangential entry tube with 1-nozzle and 2-nozzles opening plain tube is efficient and gives better results. $40 \%$ more $\mathrm{h}_{\text {expt }}$ is observed over the 1-nozzle open and $26 \%$ more $\mathrm{h}_{\text {expt }}$ is observed over 2-nozzle openings. For 3-nozzles opening, 4-nozzles openings and 5-nozzle openings value of $h_{\text {expt }}$ dominates over the plain pipe. About $35 \%, 72 \%$ and $77 \%$ augmentation in $\mathrm{h}_{\text {expt }}$ for 3-nozzles openings, 4-nozzles openings and 5nozzle openings over the plain pipe configuration is achieved respectively. For parallel flow model consecutive three openings of nozzles are required for the better results.

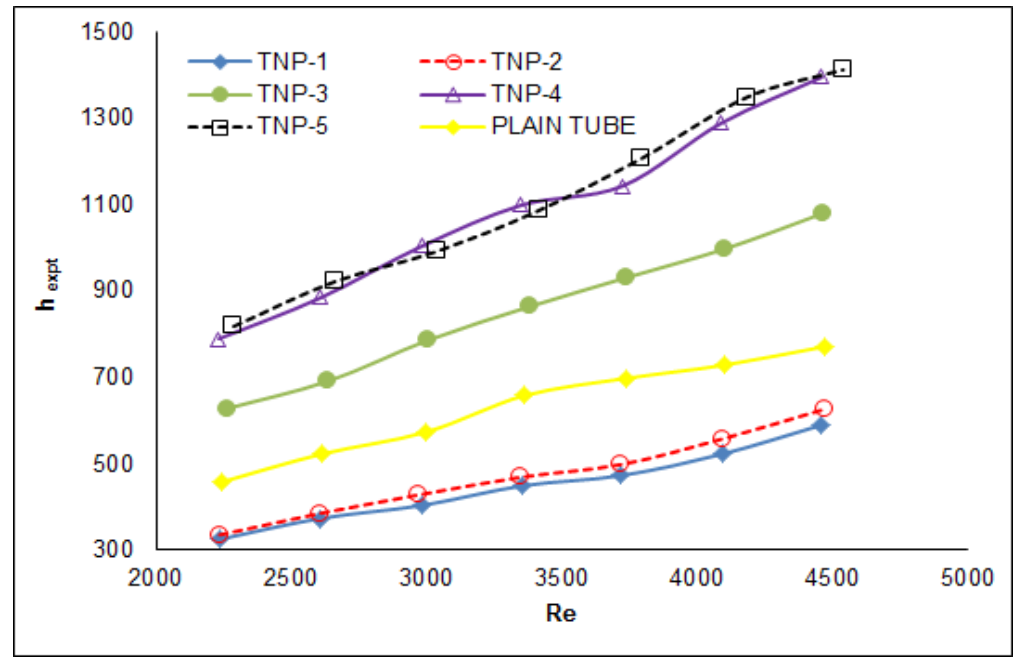

Fig.3. Variation of heat transfer coefficient with Reynolds number for plain tube and tangential injection with different nozzles openings (Parallel flow model)

The comparison between plain pipe and tangential entry pipe with different nozzle entries open for counter flow configuration as shown in Fig.4. It is seen that $h_{\text {expt }}$ increases with increase in Re for both the pipes. For plain pipe $\mathrm{h}_{\text {expt }}$ value is varies from $593-1045 \mathrm{~W} / \mathrm{m}^{2} \mathrm{~K}$ for range of Re from 2000-5000. As compared to tangential entry pipe with 1-nozzle openings and 2-nozzles openings, plain pipe configuration is efficient and 

entry of fluid

gives better results. About $38 \%$ more $\mathrm{h}_{\text {expt }}$ is observed over the 1-nozzle open and $25 \%$ more hexpt is observed over 2-nozzle openings. For 3- nozzles, 4-nozzles and 5-nozzle openings value of $\mathrm{h}_{\text {expt }}$ dominates over the plain tube value. About 19\%, $33 \%$ and $67 \%$ augmentation in hexpt for 3-nozzles, 4-nozzles and 5-nozzle openings over the plain tube is achieved respectively. For counter flow model consecutive three openings of nozzles are required for the better results. As the number of entries increases $h_{\text {expt }}$ increases with increase in Reynolds number.

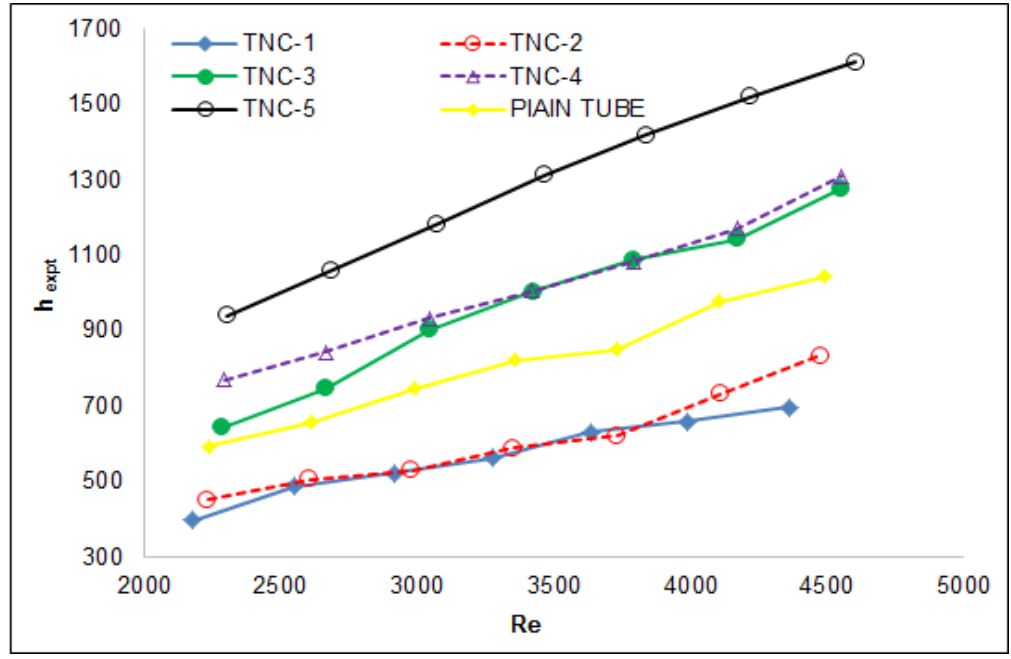

Fig.4. Variation of heat transfer coefficient with Reynolds number for plain tube and tangential injection with different nozzles openings (Counter flow model)

Fig.5. shows the performance of different tangential entries for parallel and counter flow model. From the experimental results reveals that as the number of tangential entries increases heat transfer coefficient increases with Reynolds number. It has been observed that as the number of entries increases the heat transfer coefficient increases with respect to the Reynolds number. Counter flow configuration gives the better results over the plain tube for all cases. As the number of entries increases the temperature difference goes on increasing along the flow hence the heat transfer coefficient also increases. Also the local turbulence gives the advantage of the mixing of fluid.

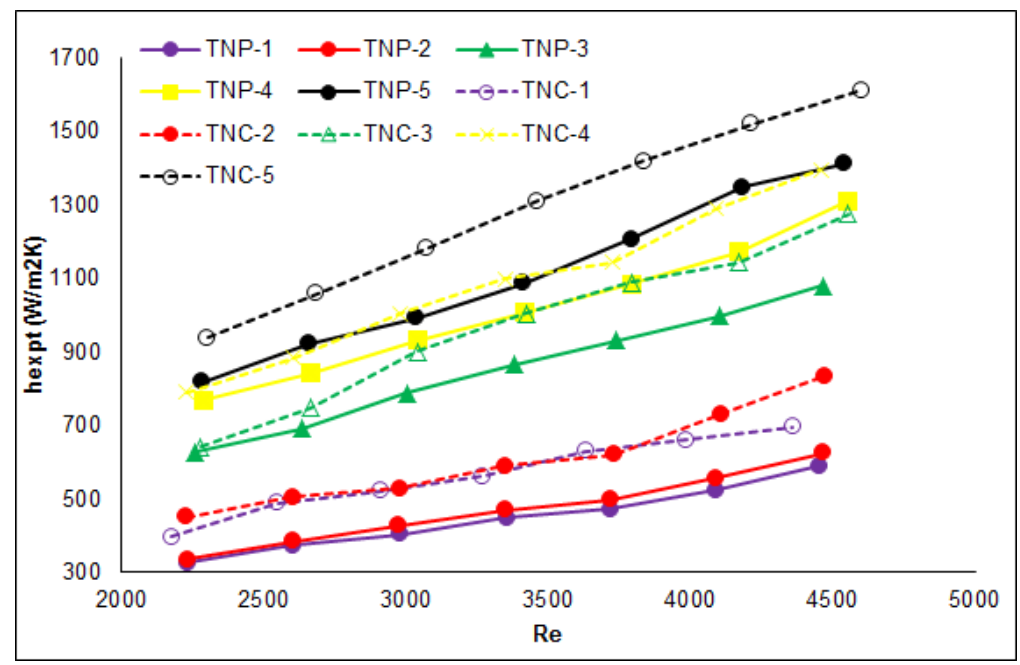

Fig.5. Variation of heat transfer coefficient with Reynolds number for tangential injection with different nozzles openings (Parallel and counter flow model) 


\section{CONCLUSIONS}

The experiment is performed for both the plain tube and tube with tangential injector entries and the conclusion is summarized as follows:

- As Reynolds number increases the heat transfer augmentation is increases for both plain tube and tangential injection configurations.

- The turbulence (swirl or vortex) in the flow is increased with every addition of tangential entry nozzles.

- The increase in heat transfer augmentation was observed about $70 \%$ with tangential entry configuration over plain pipe for counter flow configuration. The highest heat transfer augmentation was found $1613 \mathrm{~W} / \mathrm{m}^{2} \mathrm{~K}$.

- The best operating range of Reynolds number for this experimentation is 4000-4600.
TNP
tangential nozzles openings in parallel flow
TNC tangential nozzles openings in counter flow
LMTD log mean temperature difference
LPH litres per hour
$\mathrm{k} \quad$ fouling resistance

\section{REFERENCES}

[1] K Shailendhra, A. Devi, On the enhanced heat transfer in the oscillatory flow of liquid metals, Journal of Applied Fluid Mechanics, 4, 2011, 57-62.

[2] S Vahidifar, M Kahrom, Experimental study of heat transfer enhancement in a heated tube caused by wire-coil and rings, Journal of Applied Fluid Mechanics, 8 (4), 2015, 885-892.

[3] N Kumar, P Murugesan, Review of twisted tapes heat transfer enhancement, International Journal of Scientific and Engineering Research, 3 (4), 2012, 1-9.

[4] A Gupta, M Uniyal, Review of heat transfer augmentation through different passive intensifier method, IOSR Journal of Mechanical and Civil Engineering, 1 (4), 2012, 14-21.

[5] S Babar, K Devade, Heat transfer enhancement in pipe in pipe heat exchanger with swirling flow: Review, International Journal for Scientific Research \& Development, 3 (3), 2015, 990-995.

[6] Z Guo, V Dhir, Single and two phase heat transfer in tangential injection-induced swirl flow, International Journal of Heat and Fluid Flow, 10 (3), 1989, 203-210.

[7] F. Kreith, D Margolis, Heat transfer and friction in turbulent vortex flow, Applied Scientific Research, Section A, 8 (1), 1959, 457473 .

[8] M Yilmaz, O Comakli, S Yapici, Enhancement of heat transfer by turbulent decaying swirl flow, Energy Conversion and Management, 40 (13), 1999, 1365-1376.

[9] A. K. Gupta, D. G. Lilley, N. Syred, Swirl Flows (Abacus Press, Cambridge, 1984).

V Dhir, S Monica, Heat transfer enhancement using tangential injection, United State Patent, US5291943A, 1994.

[10] F Chang, V Dhir, Mechanisms of heat transfer enhancement and slow decay of swirl in tubes using tangential injection, International Journal of Heat and Fluid Flow, 16, 1995, 78-87.

[11] E Akpinar, Y Bicer, C Yildiz, D Pehlivan, Heat transfer enhancements in a concentric double pipe exchanger equipped with swirl elements, International Communications in Heat and Mass Transfer, 31 (6), 20014, 857-868.

[12] E Akpinar, Y Bicer, Investigation of heat transfer and exergy loss in a concentric double pipe exchanger equipped with swirl generator, International Journal of Thermal Sciences, 44, 2005, 598-607.

[13] G Çakmak, C Yıldız, The influence of the injectors with swirling flow generating on the heat transfer in the concentric heat exchanger, International Communications in Heat and Mass Transfer, 34, 2007, 728- 739.

[14] M. Rathore, Engineering Heat and Mass Transfer, (3rd ed. New Delhi: University science press, 2006.

[15] J Rose, Heat-transfer coefficients, Wilson plots and accuracy of thermal measurements, Experimental Thermal and Fluid Science, $28,2004,77-86$

$5^{\text {th }}$ National Conference RDME 2016, 10-11 $1^{\text {th }}$ March 2016.

M.E.S. COLLEGE OF ENGINEERING, PUNE. 411001

34 Page 